\title{
MOPSO-based optimal fuzzy logic control strategy for standalone hybrid diesel-wave energy conversion system
}

\author{
Adel Elgammal* \\ The University of Trinidad \& Tobago, Point Lisas Campus, Brechin Castle, Couva, Trinidad and Tobago
}

Received: May 14, 2014

Accepted: July 16, 2014

Online Published: August 11, 2014

DOI: $10.5430 /$ air.v3n3p11

URL: http://dx.doi.org/10.5430/air.v3n3p11

\begin{abstract}
The paper proposes a Multi-Objective Particle Swarm Optimization (MOPSO)-based Fuzzy Logic Control Proportional-IntegralDerivative (MOPSO-FLC-PID) controller for stand-alone hybrid Diesel- variable speed Wave Energy Conversion System (WECS). The optimal control performance can be achieved by tuning scaling factors of the FLC and gain parameters with Multi-Objective Particle Swarm Optimization (MOPSO) algorithm in order to improve system dynamic performance especially when nonlinearity, uncertainty and complexity of the hybrid Diesel-WECS are involved. Number of objective functions is defined to measure the performance of the proposed controllers such as minimize the transient DC and AC voltage and current ripples, minimize the DC and AC voltage and current Total Harmonic Distortion (THD), minimize the absolute total control steady state error deviations, minimize the RMS absolute voltage deviations at the DC and AC load collection buses, ensure minimal losses as well as wave energy optimal power utilization. Since some of these objective functions are conflicting, MOPSO is used to find a Pareto front from which a desired optimal operating state can be chosen. For the purpose of comparing the improvement obtained in the system dynamic performance with the application of the MOPSO-FLC-PID procedure to tune the scaling factors of the FLC and the PID controller gains, these results are compared with those obtained using traditional Ziegler-Nichols PID approach, PID type fuzzy logic controller designed with Single-Objective Particle Swarm Optimization (SOPSO-FLC-PID), traditional PID type controller optimized with SOPSO-PID and traditional PID type controller optimized with MOPSO-PID. A complete simulation model is developed for such stand-alone hybrid Diesel-WECS under variable speed operation using MATLAB Simulink environment. Simulation results show that the proposed design approaches are efficient to find the optimal scaling factors and PID gains of the controllers and therefore improves the transient performance of the hybrid Diesel-WECS over a wide range of operating conditions, sudden load change and three-phase short circuit fault.
\end{abstract}

Key Words: Stand-alone, Variable speed wave energy conversion systems, Permanent magnet synchronous Generator, Voltage and frequency control, PID self tuning, Fuzzy logic controller, Multi-objective particle swarm optimization

\section{Introduction}

Producing energy from renewable energy resources such as solar, wind, ocean, micro-hydro, biomass, etc is attracting more attention presently due to the rapid exhausting of traditional energy resources and also to control the emission of green-house gases. The wave energy seems to be one of the attractive renewable energy resources since its abundance potential and pollution-free nature, which are used for power generation for both isolated and grid connected applications $^{[1-4]}$ Wave energy conversion systems are among the most interesting and environment friendly technological solutions for the electrification of remote communities, where connection with the power grid is not feasible.

The different technologies to convert ocean wave power into electrical power include Oscillating Water Columns, marine and tidal current energy, ocean thermal energy, energy from

\footnotetext{
* Correspondence: Adel Elgammal; Email: adel_elgammal2000@yahoo.com; Address: The University of Trinidad \& Tobago UTT, Point Lisas Campus, Esperanza Road, Brechin Castle, Couva, Trinidad and Tobago
} 
salinity gradients, and cultivation of marine biomass. The Oscillating Water Columns -type wave-energy-harnessing method is considered as one of the best techniques to convert wave energy into electricity despite being far from competitive and significant scope exists for the improvement of the capacity factor using the intelligent control systems. ${ }^{[5-7]}$ There are mainly two kinds of generators, used for power generation: the asynchronous generators such as Self-Excited Induction Generators (SEIG) ${ }^{[8,9]}$ and Doubly Fed Induction Generator (DFIG) ${ }^{[10,11]}$ and synchronous generators such as diesel generator set ${ }^{[12]}$ and permanent magnet synchronous generator (PMSG). ${ }^{[13]}$ In comparison with DFIG, the usage of PMSG based wave energy conversion systems are increasing widely in power systems because of their optimal characteristics; high power factor and high efficiency operation and high generated power-weight ratio. [14-17]

Flicker or voltage fluctuations are mainly caused by the fluctuations in the wave energy, unwanted harmonics are generated due to the power electronics interface (rectifier, inverter and dc-dc converter) between the wave generator and the load, load unbalance and the control of an inverter to present the customers with a balanced supply voltage are the main Power Quality (PQ) challenges in a standalone based WECS. ${ }^{[18]}$

The control of the PMSG is achieved traditionally by vector control technique based on proportional, integral and differential controllers (PID controllers). Suitable PID controller parameters are needed for a better system performance and stability. However, the coordinated tuning of these controllers is tedious and it might be difficult due to the nonlinearity and the high complexity of the system. Therefore, it is interesting to use non-conventional control techniques, such as fuzzy logic, in order to achieve high performances and robustness. ${ }^{[19]}$ The idea of the fuzzy set theory and fuzzy control was introduced in 1965 by Zadeh in an attempt to deal with the problem of knowledge representation in an uncertain and imprecise environment. ${ }^{[20]}$

Fuzzy PID controllers have self-tuning ability and on-line adaptation to nonlinear, time varying, and uncertain systems. ${ }^{[21]}$ There are many adjustable components in a fuzzy system, such as rules, membership functions, and scaling factors. Up to now, many methods such as genetic algorithms (GA), ${ }^{[2]}$ fuzzy control, ${ }^{[23]}$ neural networks ${ }^{[24]}$ and Particle Swarm Optimization algorithm (PSO) ${ }^{[25-27]}$ have been used for scaling factor tuning.

"Particle Swarm Optimization (PSO) is an evolutionary computation optimization technique (a search method based on a natural system)" developed by Kennedy and Eberhart. ${ }^{[28-31]}$ The PSO mimics the behavior of individuals in a swarm to maximize the survival of the species. Comparing with other heuristic optimization techniques, the PSO algorithm has many advantages such as simple concept, easy implementation, robustness to control parameters, and computational efficiency. ${ }^{[27]}$ In this paper, a new structure which merges MOPSO-Based PID self-tuning and fuzzy logic control is proposed to enhance the control and adaptation ability of a stand-alone based Wave-Diesel hybrid energy conversion system. The control signal is generated by a modified PID controller with variable gains to be used under different dynamic changes and external variations and encoded in the fuzzy rule base to achieve an optimal setting with respect to a number of objective functions.

This paper is organized as follows: Section 2 presents the PMSG and WECS mathematical models description and the systems to be studied and controlled. Section 3 shows the dynamic simulations of the studied system to evaluate the performance of the proposed controllers. Finally; specific important conclusions of this paper are drawn in Section 6.

\section{Modeling of the studied system \\ 2.1 System configuration}

Figure 1 shows the proposed hybrid Diesel-Wave Energy Converter model for PMSG converting power from the wave to deliver power isolated DC and AC loads with constant frequency and constant RMS voltage for a large range of wave variation. The PMSG is coupled directly to the wave energy conversion system. The output power transfers through an AC-DC-AC stage, which consists of a diode bridge rectifier, a boost converter, and a load-side inverter, which is connected to the AC loads bus. The generated voltage from the variable waves leads to very large DC-link voltage fluctuations. A buck/boost converter is proposed to control the DC-side voltage and current to maintain the DC voltage constant, minimize the ripples in the DC link current and capture maximum wave energy over the whole wave level range. Also, the system is connected to the ACbus load though a vector controlled Pulse Width Modulated (PWM) IGBT-inverter to to invert the constant DC power into AC power for the desired single-phase and three-phase loads and control the amplitude and frequency of the supply voltage. The diesel motor is used for the non-grid-connected wave energy conversion system according to time span in the low wave level periods. The vector controlled PWM voltage source IGBT-inverter is proposed.

\subsection{DC-Side converter control}

The AC power output from the generator is converted into DC power through diode rectifier circuits, and it is boosted by the boost chopper circuit. The DC-DC converter was designed around a low cost single switching device buck/boost chopper as shown in Figure 2. The basic idea of the proposed control strategy of the switch mode rectifier is to control the duty cycle of the DC chopper circuit based on the measured samples of the voltage and current signals $\left(v_{d}\right.$ and $i_{d}$ ) in order to make the output voltage keep constant. The control algorithm includes the following steps: measure the output DC voltage and current $\left(v_{d}\right.$ and $\left.i_{d}\right)$, set the refer- 
ence DC voltage $\left(v_{d}\right)$. In the first loop, the actual signal of the dc-link voltage $\left(v_{d}\right)$ is compared with the dc-link reference voltage $\left(v_{d}^{*}\right)$ to form the DC voltage error signal, which is multiplied by a scaling factor $\left(\alpha_{v}\right)$ and passed through a summation block to be added to the dc current error. In the second loop, the actual signal of the dc-link current $\left(i_{d}(k)\right)$ is compared with the delayed dc-link current $\left(i_{d}(k+1)\right)$ to form the DC current error signal, which is multiplied by a scaling factor $\left(\alpha_{i}\right)$ and passed through a summation block to be added to the dc voltage error. The total dc error is passed through the FLC-based PID controller to generate the reference switch signals for DC chopper.

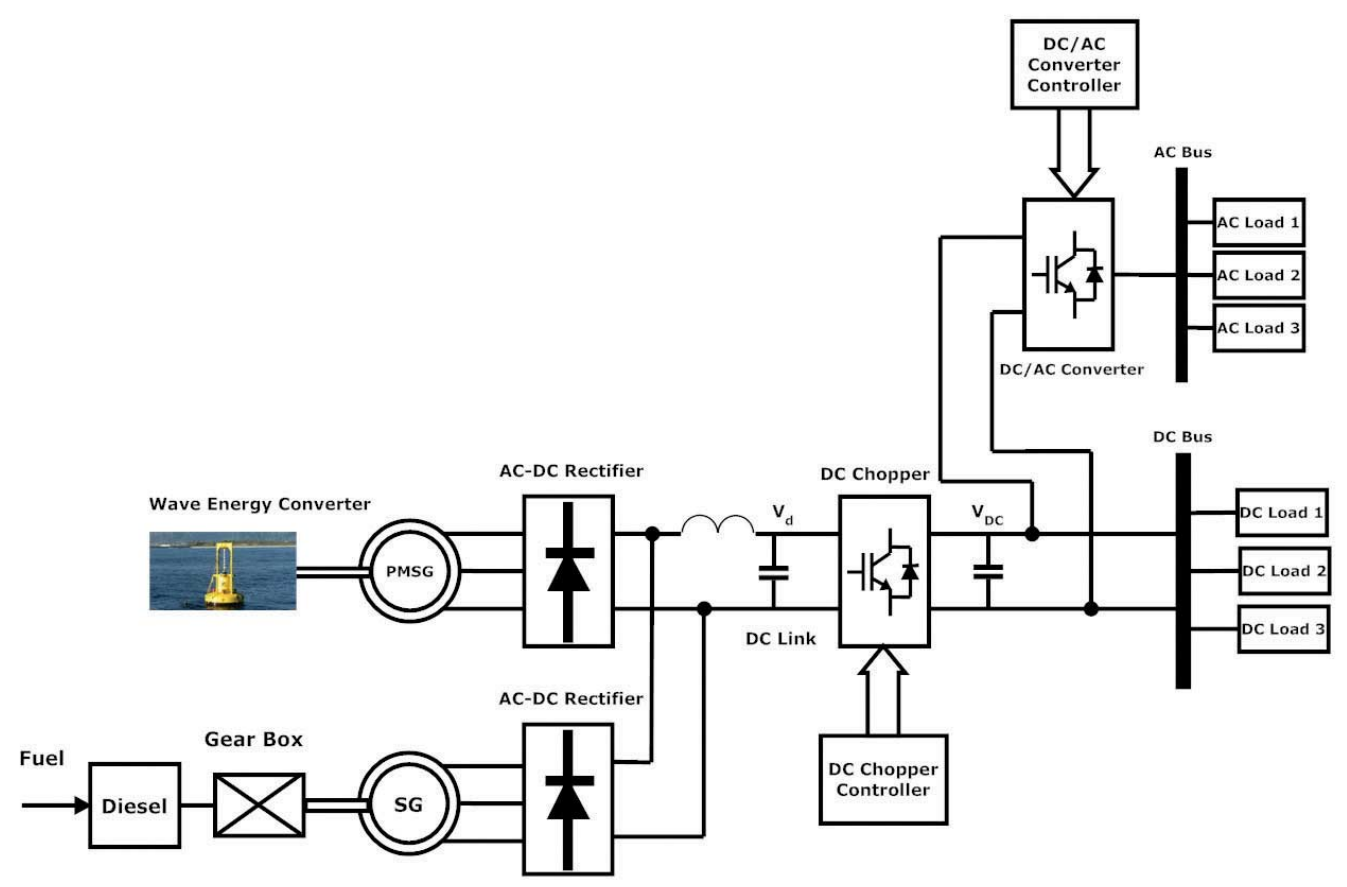

Figure 1: Schematic representation of the proposed hybrid Diesel-Wave energy converter with PMSG

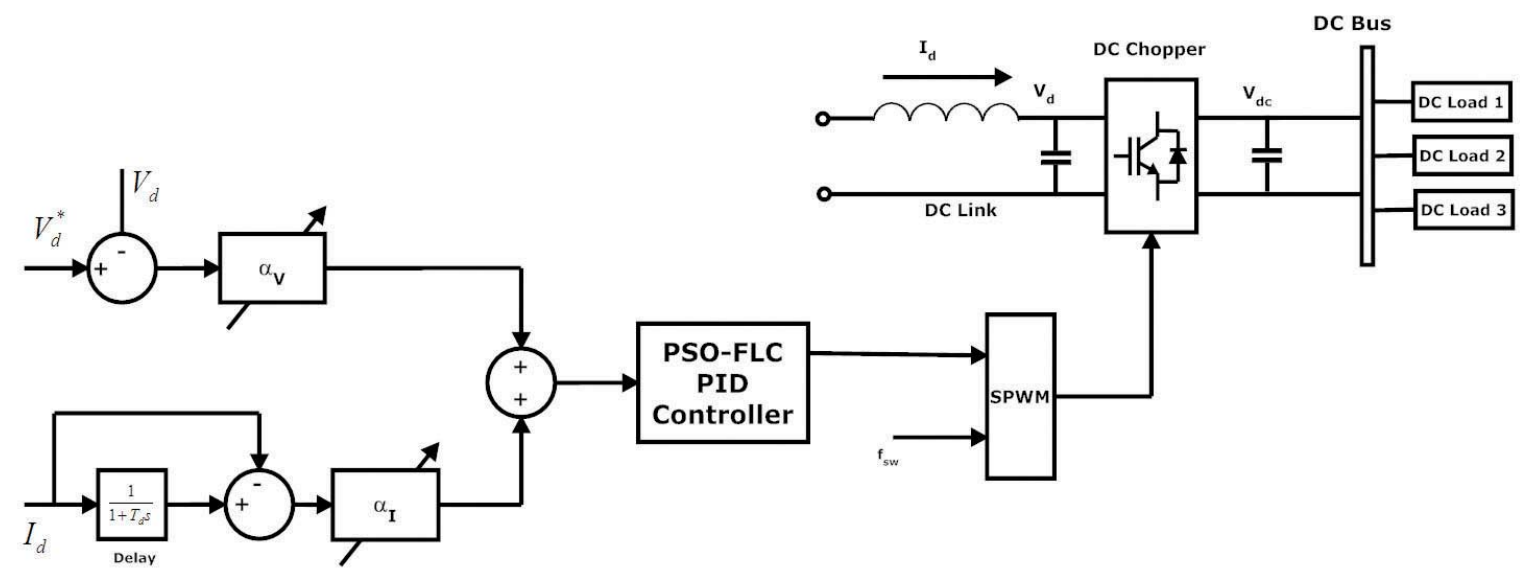

Figure 2: Schematic of control strategy for DC load bus Converter

\subsection{AC-Side converter control}

Figure 3 shows the overall control scheme of the AC-side inverter. The control of the AC-side converter are organized in two loops; a quadrature voltage loop, which controls the quadrature current component and DC-link voltage control loop that controls the dc-link voltage. This implies that the dc-link voltage control loop has to act on the $\mathrm{d}$ component

Published by Sciedu Press of the current. DC-link voltage controller is used to stabilize the dc link voltage. The dc-voltage PID controller maintains the dc voltage to the reference value. The actual signal of the dc-link voltage $\left(v_{d c}\right)$ is compared with its command $\left(v_{d c}^{*}\right)$ to form the error signal, which is passed through the PID controller to generate the reference signals for the $d$-axis current component $\left(i_{d s}^{*}\right)$. In the second loop of the 
AC-load side converter, the quadrature voltage component is compared with the quadrature reference voltage to generate the error signal which is passed through the PID controller to generate the reference signal for the $q$-axis current component $\left(i_{q s}^{*}\right)$. The instantaneous three-phase reference currents $\left(i_{a}^{*}, i_{b}^{*}, i_{c}^{*}\right)$ are generated by transforming the reference d-q components $i_{d s}^{*}$ and $i_{q s}^{*}$ in the stator-flux oriented reference frame. The actual three-phase currents signals $\left(i_{a}\right.$, $\left.i_{b}, i_{c}\right)$ are then compared with their reference signals $\left(i_{a}^{*}, i_{b}^{*}\right.$, $\left.i_{c}^{*}\right)$ to generate the error signals, which are passed through the hysteresis current controller to form the switching signals. They are then used by the PWM module to generate the IGBT gate control signals to drive the IGBT converter.

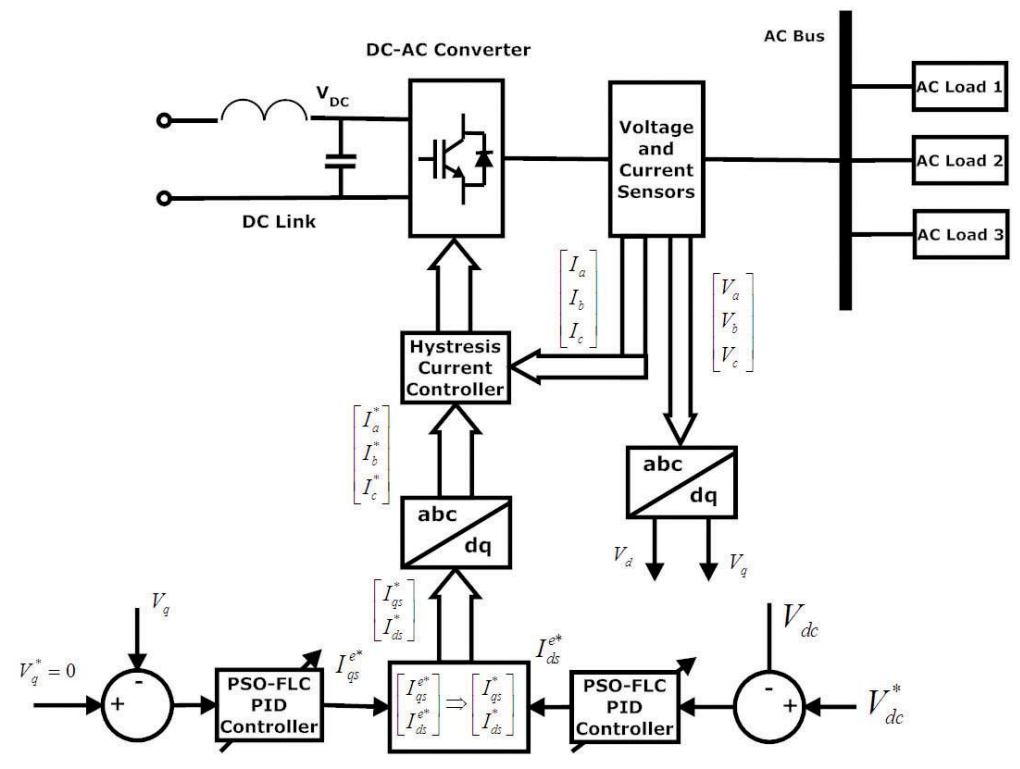

Figure 3: Schematic of control strategy for AC load bus inverter

\subsection{Fuzzy logic control-based PID controller (FLC PID) structure}

The overall design procedure of the fuzzy PID controller realized by means of PSO is illustrated in Figure 4. It has the basic PID structure, but the PID parameter settings are generated by fuzzy inference, which provides a nonlinear mapping from the error signal e (difference between system output and reference signal and defined as $\mathrm{e}(\mathrm{k})=$ Reference $(\mathrm{k})$ - Output (k)) to the PID gains $K p, K i$ and $K d . \Delta e$ is the first-order difference of error signal and defined as $\Delta e(\mathrm{k})=$ $\mathrm{e}(\mathrm{k})-\mathrm{e}(\mathrm{k}-1)$. Note that the input variables to the fuzzy controller ( $\mathrm{E}$ and $\Delta E$ ) are transformed by multiplying the error (e) and the first-order difference of error signal $(\Delta e)$ by the scaling factors $\beta_{1}$ and $\beta_{2}$ whose role is to allow the fuzzy controller to "see" the external world to be controlled. The dynamic behavior of this PID-type FC structure is strongly dependent on the scaling factors $\beta_{1}$ and $\beta_{2}$ difficult and delicate to tune. If the controllers are tuned properly, it is possible to improve the two converter's performance during the transient disturbances. The choice of the adequate values for the scaling factors of each PID-type FC structure is often done by a trials-errors hard procedure. Therefore, tuning controllers is tedious and it is difficult to achieve a set of optimal parameters manually and becomes difficult and delicate without a systematic design method. To deal with these difficulties, the optimization of these scaling factors $\beta_{1}$ and $\beta_{2}$ tuned based on PSO because the control parameters have an effect on the performance of controller. The MOPSO algorithm is applied to find the optimal parameters of the controllers automatically. In the DC-side and the AC-side control loops, there are three PID controllers and each of them has a proportional gain, an integral, a differential gains and two scaling factors. The objective of the MOPSO is to find the optimal parameters of the PID controllers, namely, three proportional gains $\left(K_{P 1}, K_{P 2}\right.$, and $\left.K_{P 3}\right)$, three integral gains $\left(K_{11}, K_{12}\right.$, and $\left.K_{13}\right)$ and three differential gains $\left(K_{D 1}, K_{D 2}\right.$, and $\left.K_{D 3}\right)$ to optimize the proposed fitness functions. The set of rules which define the relation between the input and output of fuzzy controller can be found using the available knowledge in the area of designing the system. These rules are defined using the linguistic variables. The membership functions for the inputs and outputs for the fuzzy controller are shown in figures 5 and 6. Tables (1), (2) and (3) show the control rules that used for fuzzy self-tuning of PID controller. Where, NB: negative big, NM: negative medium, NS: negative small, ZE: zero, PS: positive small, PM: positive medium and PB: positive big. VB: very big, B: big, MB: medium big, ZE: zero, M: medium, S: small, MS: medium small. 


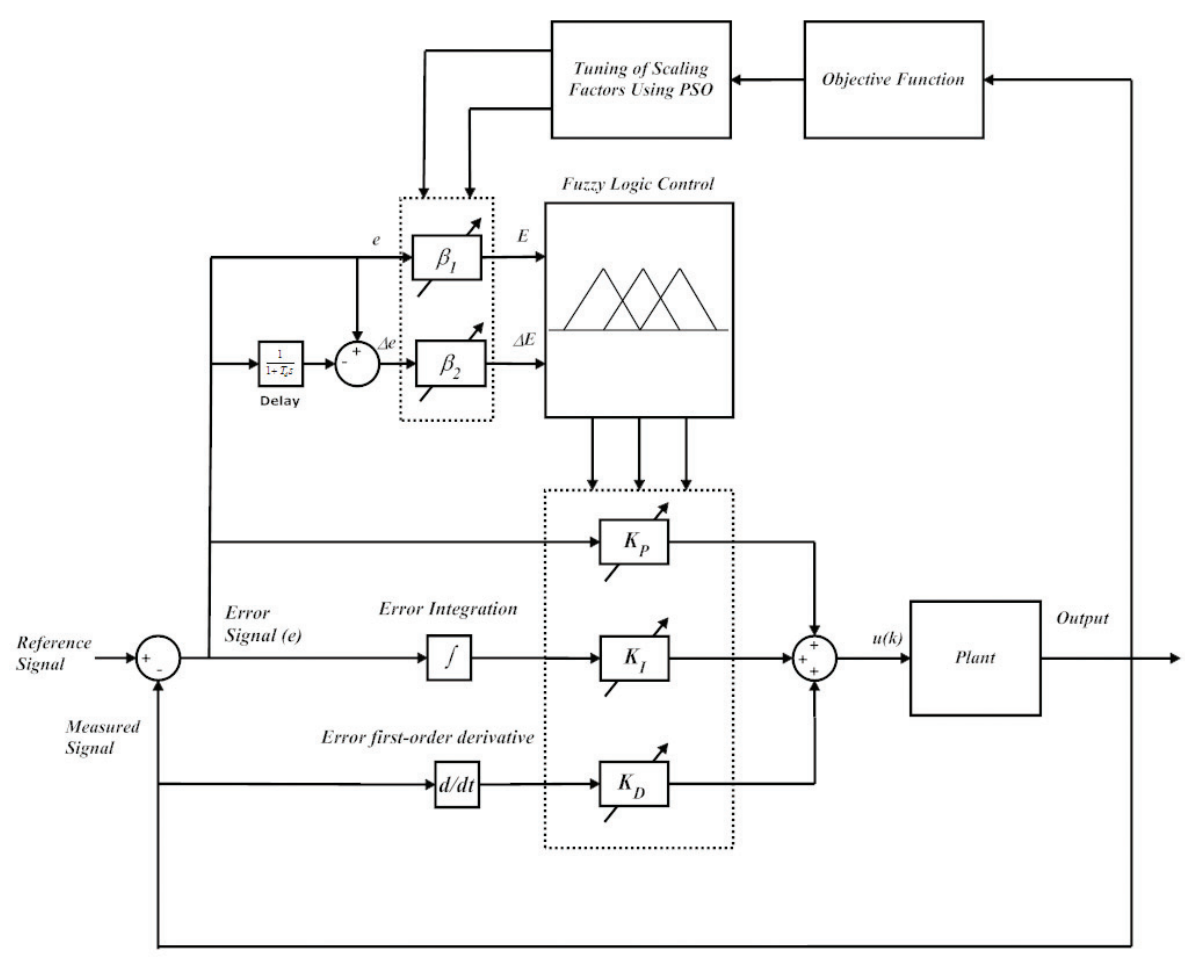

Figure 4: Schematic of the PSO-Based scaling factors -tuning of the fuzzy PID controller

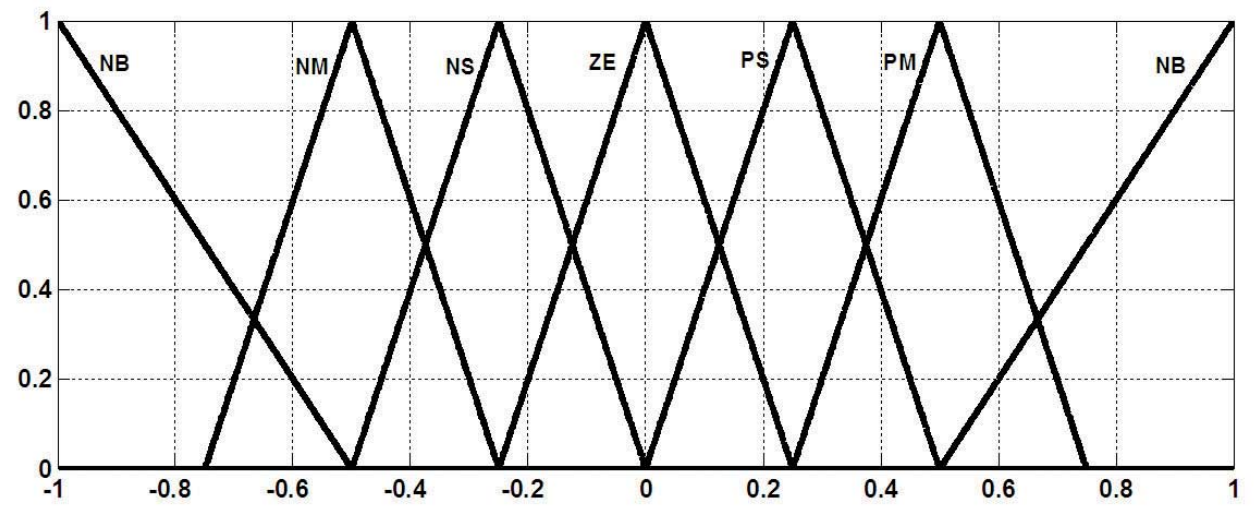

Figure 5: Membership functions of inputs (E, $\Delta \mathrm{E}$ )

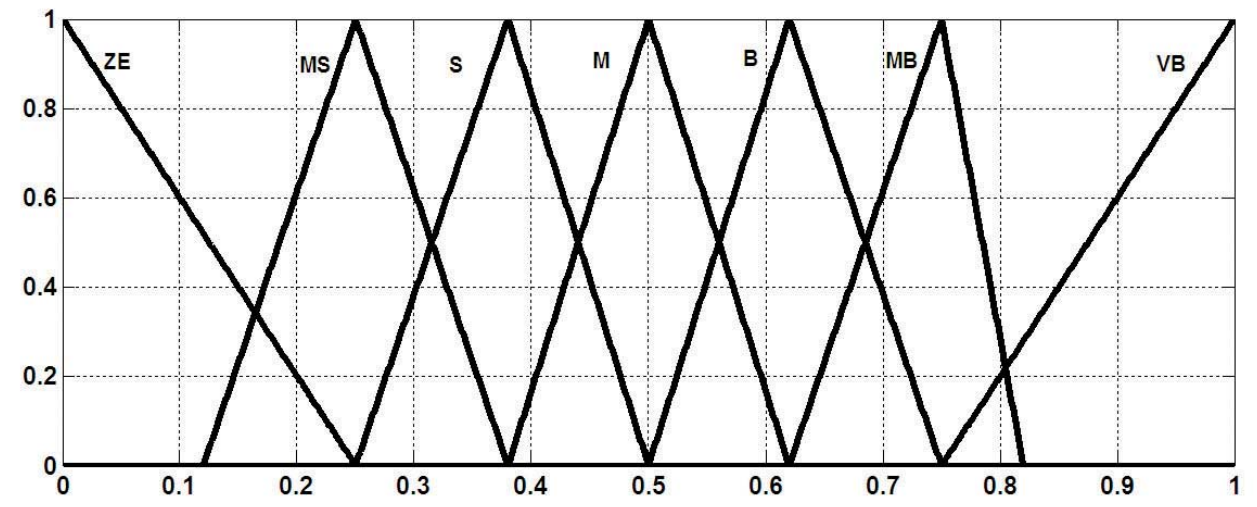

Figure 6: Membership functions of outputs $\left(K_{P}, K_{I}\right.$, and $\left.K_{D}\right)$ 
Table 1: Rule bases for tuning $K_{P}$

\begin{tabular}{llllllll}
\hline $\mathbf{\Delta e} / \mathbf{e}$ & NB & NM & NS & ZE & PS & PM & PB \\
\hline NB & VB & VB & VB & VB & VB & VB & VB \\
NM & VB & VB & MB & MB & MB & VB & VB \\
NS & B & B & B & B & MB & MB & VB \\
ZE & ZE & S & ZE & MS & S & S & S \\
PS & B & B & B & B & MB & MB & VB \\
PM & VB & VB & MB & MB & MB & VB & VB \\
PB & VB & VB & VB & VB & VB & VB & VB \\
\hline
\end{tabular}

Table 2: Rule bases for tuning $K_{I}$

\begin{tabular}{llllllll}
\hline $\mathbf{a} / \mathbf{e}$ & NB & NM & NS & ZE & PS & PM & PB \\
\hline NB & M & M & M & M & M & M & M \\
NM & MS & MS & MS & MS & MS & MS & MS \\
NS & S & S & S & S & S & S & S \\
ZE & MS & MS & MS & ZE & MS & MS & MS \\
PS & S & S & S & S & S & S & S \\
PM & MS & MS & MS & MS & MS & MS & MS \\
PB & M & M & M & M & M & M & M \\
\hline
\end{tabular}

\section{Digital simulation results}

The proposed MOPSO strategy has been tested for validation using the PMSG whose ratings are given in the Appendix. The wave model of Fig. 7 is adopted to generate a specific power reference and validate the good power tracking performances and therefore confirm the effectiveness of the proposed control strategy based on MOPSO. To compare the improvement obtained in the system dynamic performance with the application of the MOPSO procedure to design the scaling factors of the fuzzy logic-based PID controller, these results is compared with those obtained using the SOPSO for tuning fuzzy PID controllers, standard MOPSO-based for tuning PID controller, SOPSO-based for tuning PID controller and Ziegler-Nichols PID approaches.

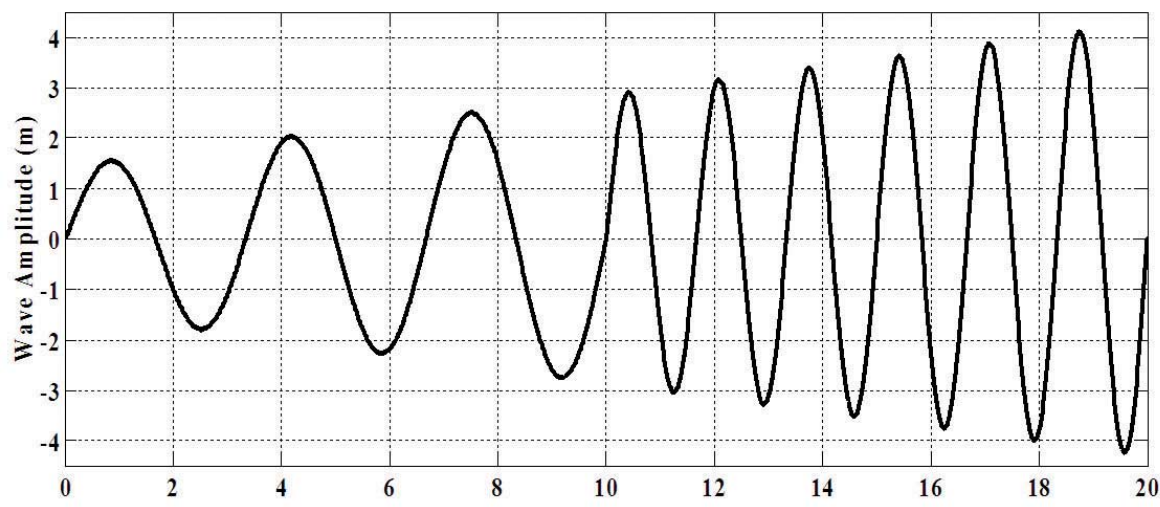

Figure 7: The wave model

The quality of the gains adjustment is measured by an index that represents the weighted sum of the Normalised Mean Square Error (NMSE) deviations between output plant variables and desired values. This way the objective to improve the system performance during the transient disturbances will be obtained by specifying optimal values for the controller gains. The NMSE deviations between output plant variables and desired values are defined as:

$$
J_{1}=\operatorname{minimize}\left\{N M S E_{V D C}\right\}=\operatorname{minimize}\left\{\frac{\sum\left(V_{D C}-V_{D C-r e f}\right)^{2}}{\sum\left(V_{D C}-r e f\right)^{2}}\right\}
$$




$$
\begin{aligned}
& J_{2}=\operatorname{minimize}\left\{N M S E_{I D C}\right\}=\operatorname{minimize}\left\{\frac{\sum\left(I_{D C}-I_{D C-r e f}\right)^{2}}{\sum\left(I_{D C-r e f}\right)^{2}}\right\} \\
& J_{3}=\operatorname{minimize}\left\{N M S E_{V A C}\right\}=\operatorname{minimize}\left\{\frac{\sum\left(V_{A C}-V_{A C-r e f}\right)^{2}}{\sum\left(V_{A C-r e f}\right)^{2}}\right\} \\
& J_{4}=\operatorname{minimize}\left\{T H D_{V D C}\right\}=\operatorname{minimize}\left\{\frac{\sqrt{\sum_{n=2}^{\infty} V_{d n}^{2}}}{V_{d 1}}\right\}
\end{aligned}
$$

Where $T H D_{V D C}$ is the Total Harmonic Distortion (THD) of the DC bus voltage and $V_{d 1}, V_{d 2}, V_{d 3}, V_{d 4}, V_{d 5} \ldots$ are the DC bus voltages at their respective harmonics. The objective is to reduce the THD and fluctuations/variations in the Common DC collection system by limiting DC bus $V_{d}$ tran- sients and ripple content. The THD of a waveform is calculated by taking the square root of the addition of the squares of the harmonic currents, and dividing them by the fundamental component.

$$
J_{5}=\operatorname{minimize}\left\{T H D_{I D C}\right\}=\operatorname{minimize}\left\{\frac{\sqrt{\sum_{n=2}^{\infty} I_{d n}^{2}}}{I_{d 1}}\right\}
$$

Where $T H D_{I D C}$ is the THD of the DC bus current and $V_{d 1}, V_{d 2}, V_{d 3}, V_{d 4}, V_{d 5} \ldots$ are the $\mathrm{DC}$ bus currents at their respective harmonics.

The Single Objective Optimization and Multi Objective Optimization strategies are used to solve this complex optimality search problem. A single global optimal solution is obtained by optimizing a single aggregate objective function with weighted single objective parameters scaled by a number of weighting factors. The weighted single objective function $\left(J_{0}\right)$ combines several objective functions using specified or selected weighting factors as follows:

$$
J_{0}=\int_{0}^{t}\left(\alpha_{1} J_{1}+\alpha_{2} J_{2}+\alpha_{3} J_{3}+\alpha_{4} J_{4}+\alpha_{5} J_{5}\right) d t
$$

Where $\alpha_{1}=0.2, \alpha_{2}=0.2, \alpha_{3}=0.2, \alpha_{4}=0.2$ and $\alpha_{5}=0.2$, are selected weighting factors. $J_{1}, J_{2}, J_{3}, J_{4}$ and $J_{5}$ are the selected objective functions. On the other hand, the main ob- jective of the Multi-Objective optimization strategy is finding the set of acceptable Optimal Solutions (Pareto front). In the calculation of the optimal gains by the MOPSO-FLCPID procedure, the objective is to improve the overall dynamic performance of the hybrid Diesel-WECS renewable energy system when it is subjected to severe electrical load disturbances and faults in the electrical network. The dynamic simulations were carried out for a load change by $50 \%$ and a three phase short circuit next to the $\mathrm{AC}$ bus at time $\mathrm{t}=0.2 \mathrm{~s}$, lasting for $0.2 \mathrm{~s}$. The gain values for different approaches are presented in tables 4, 5 and 6. Table 7 shows the system behavior comparison using the SOPSOPID, MOPSO-PID, SOPSO-FLC-PID and MOPSO-FLCPID based Self tuned controllers. The oscillations and the maximum overshoot of the dc-link voltage and current, when applying the MOPSO-FLC-PID are slightly smaller than when applying the MOPSO-PID and SOPSO-PID, as shown in Figures 8-11.

Table 4: The optimal parameters of the DC Load Bus in different approaches

\begin{tabular}{llllll}
\hline & Ziegler-Nichols PID & SOPSO PID & MOPSO PID & SOPSO Fuzzy PID & MOPSO Fuzzy PID \\
\hline $\mathrm{K}_{\mathrm{P}}$ & 3.9668 & 5.2922 & 6.6309 & 4.9552 & 9.9871 \\
$\mathrm{~K}_{\mathrm{I}}$ & 1.1878 & 0.2885 & 0.4681 & 0.7744 & 0.6187 \\
$\mathrm{~K}_{\mathrm{D}}$ & 0.2417 & 0.3033 & 0.2114 & 0.8798 & 0.3536 \\
$\alpha_{\mathrm{v}}$ & 1.0786 & 1.8861 & 2.3159 & 1.3774 & 1.7973 \\
$\alpha_{\mathrm{I}}$ & 0.8552 & 0.7853 & 0.8890 & 0.8517 & 0.3655 \\
$\beta_{1}$ & 2.6248 & 2.3728 & 1.2406 & 2.1329 & 1.2123 \\
$\beta_{2}$ & 2.0101 & 1.5885 & 1.7914 & 1.9618 & 2.0676 \\
\hline
\end{tabular}


However, the dc-link voltage oscillations in both designs, with and without FLC do not affect the continuous operation of the hybrid Diesel-WECS and consequently improving the PMSG dynamic performance. Figures 12-14 show the AC-side voltage, current and power factor dynamic behaviors for constant load, sudden load change and 3-phase short circuit fault. A reduction in the over-current can easily be observed, when the gains are adjusted by the MOPSO-FLC_PID as compared with those adjusted via other approaches, consequently contributes towards maintaining the converter in operation during the fault period. In addition, it is observed that with the use of gains ob- tained without FLC the terminal voltage presents deeper sag as compared with those controllers with gains adjusted by the proposed MOPSO-FLC_PID procedure. Figures 15-18 illustrate the total harmonic distortion measurement in the three phase grid voltage waveforms, three phase grid current waveforms, three phase rotor voltage waveforms and three phase rotor current wave forms. The system produces less than $10 \%$ voltage and current THD which is compliant to IEC 6100-3-2. The simulation results demonstrate that the MOPSO-FLC-PID controller works very well and shows very good dynamic and steady state performance.

Table 5: The optimal parameters of the AC Load Bus-quadrature axis loop in different approaches

\begin{tabular}{llllll}
\hline & Ziegler-Nichols PID & SOPSO PID & MOPSO PID & SOPSO Fuzzy PID & MOPSO Fuzzy PID \\
\hline $\mathrm{K}_{\mathrm{P}}$ & 6.5376 & 8.9092 & 7.4407 & 6.1767 & 9.3993 \\
$\mathrm{~K}_{\mathrm{I}}$ & 0.9417 & 1.3416 & 1.0002 & 1.5944 & 1.8651 \\
$\mathrm{~K}_{\mathrm{D}}$ & 0.7905 & 0.9875 & 0.2992 & 0.5492 & 0.2867 \\
$\beta_{1}$ & 1.1504 & 1.9781 & 1.0472 & 1.7672 & 1.8990 \\
$\beta_{2}$ & 1.0372 & 1.3054 & 1.0987 & 0.8292 & 1.6793 \\
\hline
\end{tabular}

Table 6: The optimal parameters of the AC Load Bus-Direct axis loop in different approaches

\begin{tabular}{llllll}
\hline & Ziegler-Nichols PID & SOPSO PID & MOPSO PID & SOPSO Fuzzy PID & MOPSO Fuzzy PID \\
\hline $\mathrm{K}_{\mathrm{P}}$ & 9.7868 & 6.8197 & 8.1815 & 5.1859 & 4.3239 \\
$\mathrm{~K}_{\mathrm{I}}$ & 1.1269 & 0.4243 & 1.1755 & 0.7297 & 1.2531 \\
$\mathrm{~K}_{\mathrm{D}}$ & 1.0047 & 0.7145 & 0.2244 & 0.4899 & 0.8347 \\
$\beta_{1}$ & 0.7109 & 1.2165 & 0.4987 & 1.0033 & 0.3317 \\
$\beta_{2}$ & 0.5962 & 0.9673 & 6.5961 & 0.5380 & 0.7339 \\
\hline
\end{tabular}

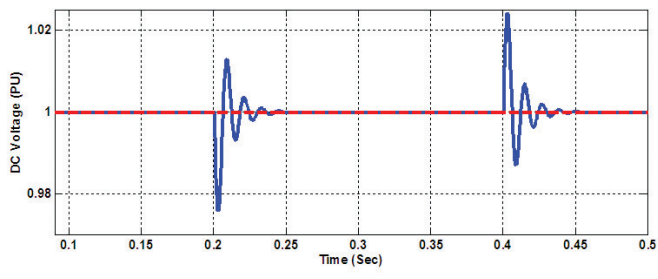

(a)

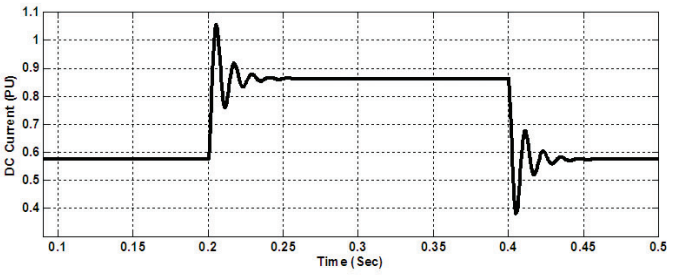

(b)

Figure 8: DC link Voltage and current responses-based SOPSO-PID controller when load is increased by $50 \%$

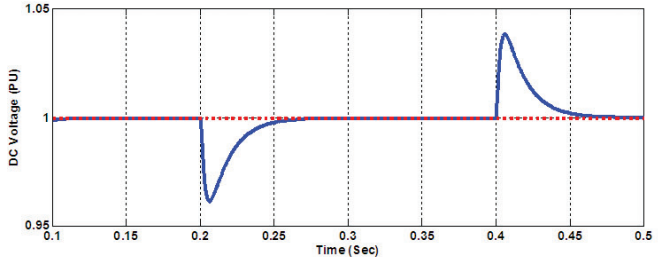

(a)

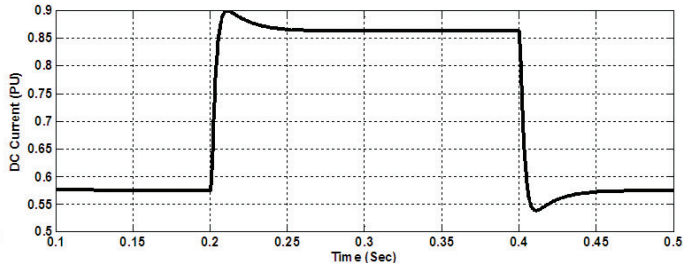

(b)

Figure 9: DC link Voltage and current responses-based MOPSO-PID Controller when load is increased by $50 \%$. 


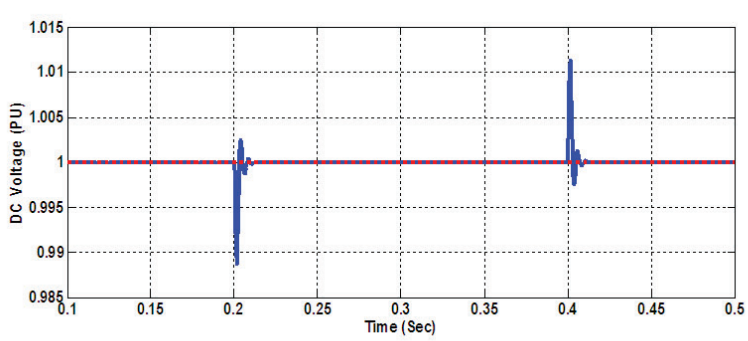

(a)

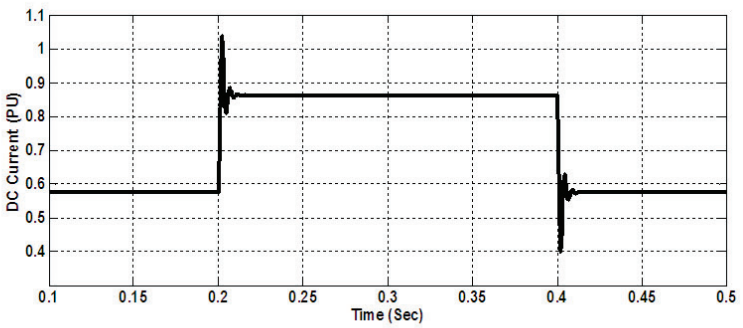

(b)

Figure 10: DC link Voltage and current responses-based SOPSO-FLC-PID Controller when load is increased by 50\%.

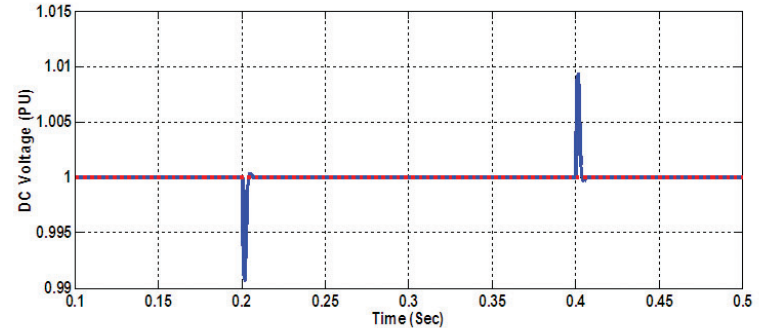

(a)

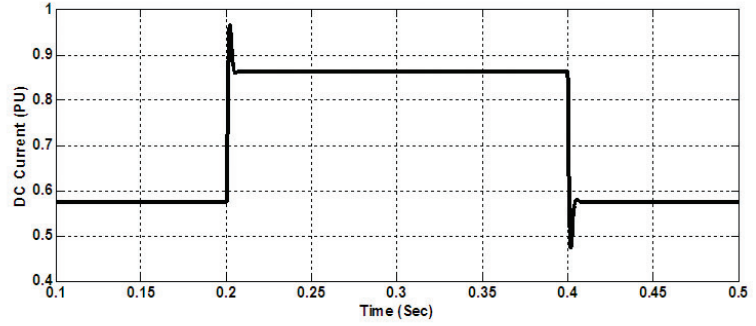

(b)

Figure 11: DC link Voltage and current responses-based MOPSO-FLC-PID Controller when load is increased by 50\%.

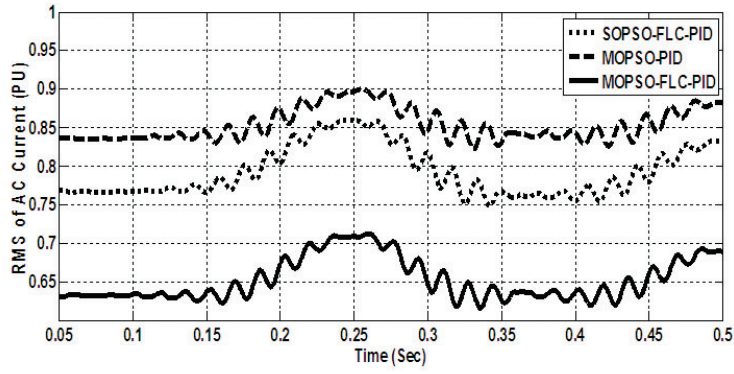

(a)

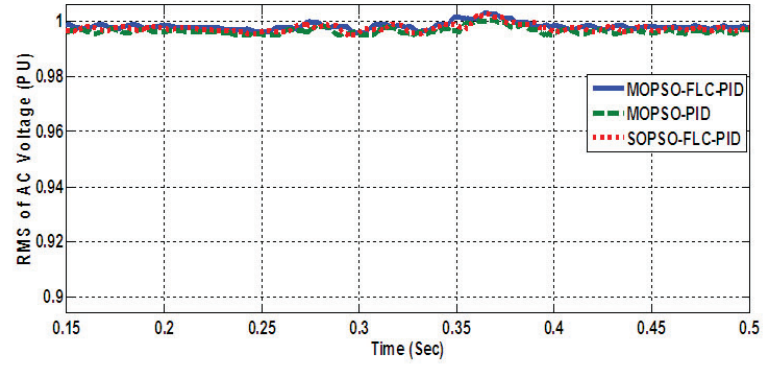

(b)

Figure 12: AC Voltage and Current responses at a constant load.

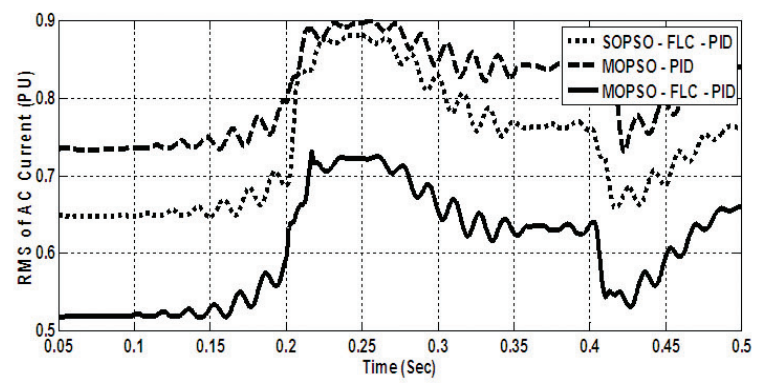

(a)

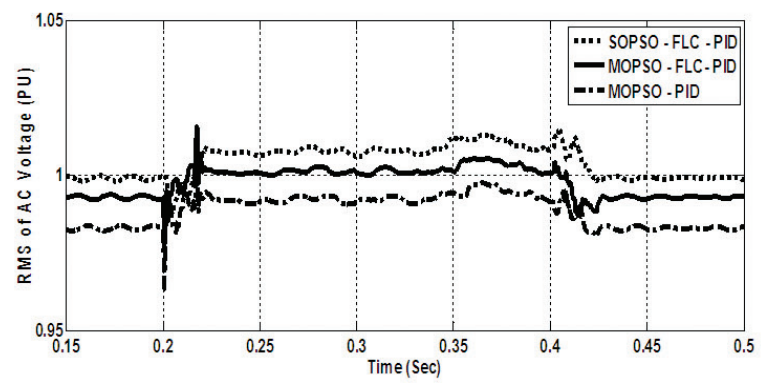

(b)

Figure 13: AC Voltage and Current responses when the load suddenly increased by $50 \%$. 


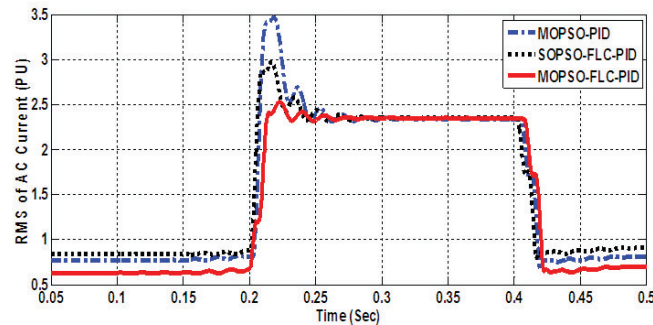

(a)

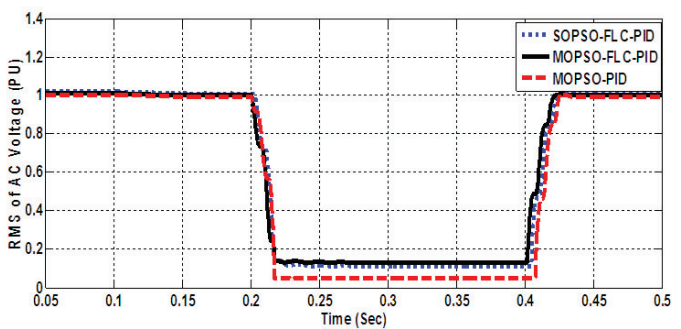

(b)

Figure 14: AC Voltage and Current responses at a short circuit fault.
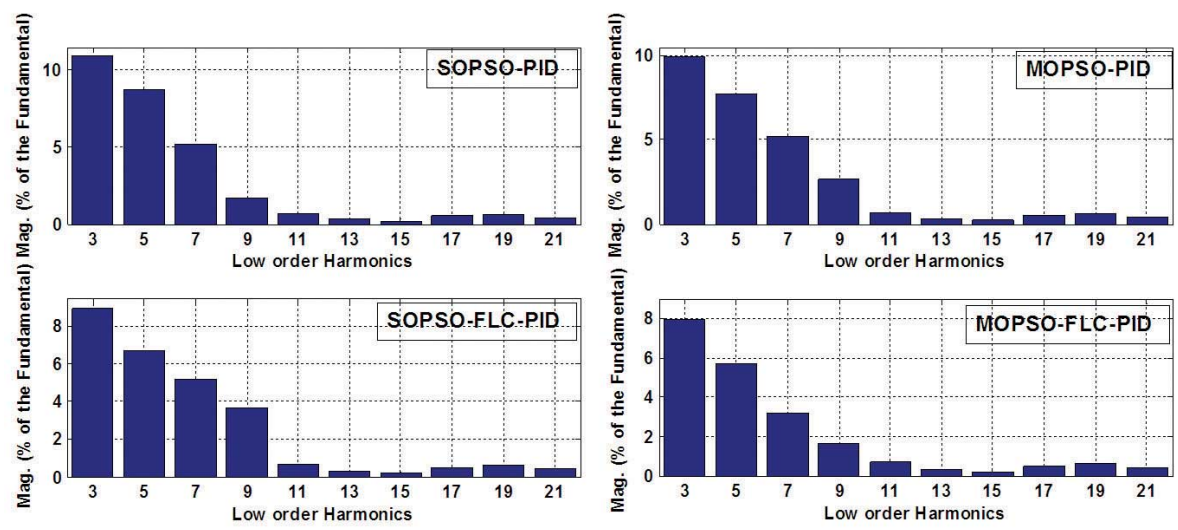

Figure 15: THD level for DC Side voltage waveform.
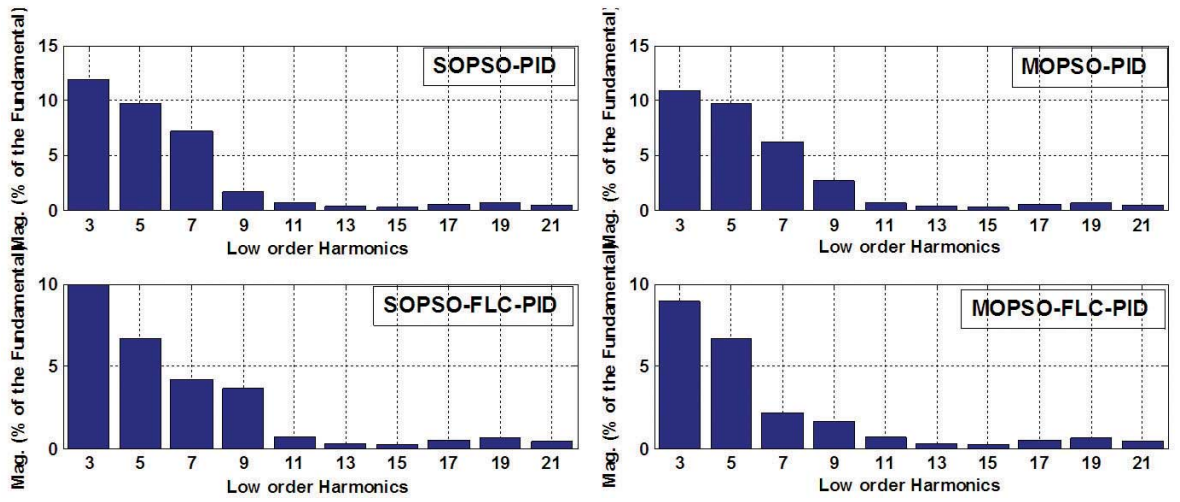

Figure 16: THD level for DC Side current waveform.
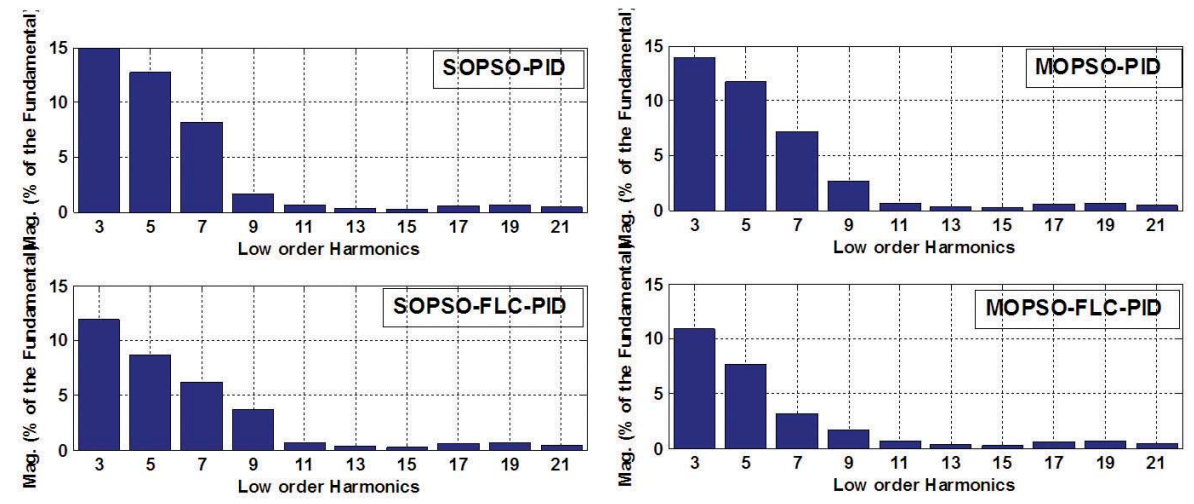

Figure 17: THD level for AC-Side voltage waveform. 
Table 7: System dynamic behavior comparison in different approaches

\begin{tabular}{|c|c|c|c|c|c|}
\hline & $\begin{array}{l}\text { Ziegler-Nichols } \\
\text { PID }\end{array}$ & SOPSO PID & MOPSO PID & $\begin{array}{l}\text { SOPSO Fuzzy } \\
\text { PID }\end{array}$ & $\begin{array}{l}\text { MOPSO Fuzzy } \\
\text { PID }\end{array}$ \\
\hline RMS AC Voltage (PU) & 0.9895 & 0.9950 & 0.9966 & 0.9987 & 0.9994 \\
\hline RMS AC Current (PU) & 0.6516 & 0.5991 & 0.5895 & 0.5440 & 0.4076 \\
\hline RMS DC Voltage (PU) & 0.9986 & 0.9991 & 0.9992 & 0.9996 & 0.9999 \\
\hline RMS DC Current (PU) & 0.5527 & 0.5326 & 0.4846 & 0.4253 & 0.4184 \\
\hline $\begin{array}{l}\text { Maximum Transient AC Voltage } \\
\text { Over/Under Shoot (PU) }\end{array}$ & 0.08379 & 0.08012 & 0.07830 & 0.07127 & 0.0686 \\
\hline $\begin{array}{l}\text { Maximum Transient AC Current - } \\
\text { Over/Under Shoot (PU) }\end{array}$ & 0.09691 & 0.08819 & 0.08518 & 0.07615 & 0.05313 \\
\hline $\begin{array}{l}\text { Maximum Transient DC Voltage } \\
\text { Over/Under Shoot (PU) }\end{array}$ & 0.07228 & 0.06692 & 0.05904 & 0.05088 & 0.04251 \\
\hline $\begin{array}{l}\text { Maximum Transient DC Current - } \\
\text { Over/Under Shoot (PU) }\end{array}$ & 0.07479 & 0.06904 & 0.06171 & 0.04229 & 0.04056 \\
\hline $\mathrm{NMSE}_{V D C}$ & 0.009427 & 0.008689 & 0.007653 & 0.006642 & 0.006110 \\
\hline $\mathrm{NMSE}_{\text {IDC }}$ & 0.000377 & 0.000307 & 0.000244 & 0.000195 & 0.000188 \\
\hline $\mathrm{NMSE}_{\mathrm{VAC}}$ & 0.009831 & 0.005916 & 0.005827 & 0.004709 & 0.004235 \\
\hline $\mathrm{NMSE}_{f}$ & 0.009015 & 0.007564 & 0.007302 & 0.006959 & 0.006908 \\
\hline $\mathrm{THD}_{\mathrm{VDC}}(\%)$ & 0.09011 & 0.08555 & 0.06439 & 0.05999 & 0.04665 \\
\hline $\mathrm{THD}_{\text {IDC }}(\%)$ & 0.09663 & 0.09448 & 0.08841 & 0.08385 & 0.06537 \\
\hline $\mathrm{THD}_{\mathrm{VAC}}(\%)$ & 0.06391 & 0.05763 & 0.04078 & 0.00336 & 0.02810 \\
\hline $\mathrm{THD}_{\mathrm{IAC}}(\%)$ & 0.06981 & 0.06509 & 0.05063 & 0.00588 & 0.04401 \\
\hline System Efficiency (PU) & 0.7265 & 0.7283 & 0.7397 & 0.7396 & 0.7471 \\
\hline System Power Factor & 0.9781 & 0.9820 & 0.9978 & 0.9909 & 0.9994 \\
\hline
\end{tabular}

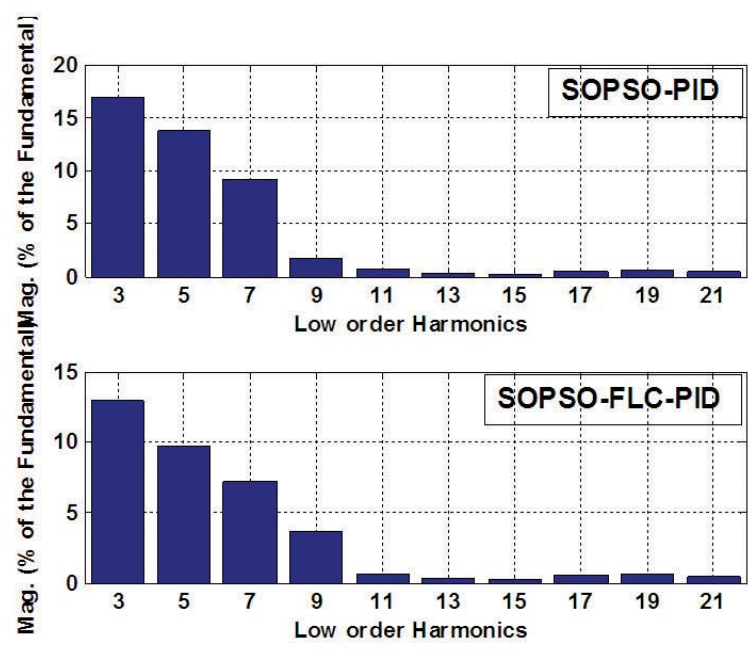

Figure 18: THD level for AC-Side current waveform.

\section{Conclusion}

In this paper, a control strategy based on MOPSO for tuning the scaling factors of the FLC is proposed and successfully applied to stand-alone hybrid Diesel- variable speed WECS. The AC load side PWM inverter is controlled using vector control scheme to maintain the amplitude and frequency of the inverter output voltage quite well at constant load and under varying load conditions, fluctuating waves and threephase short circuit fault. The DC-DC converter controller

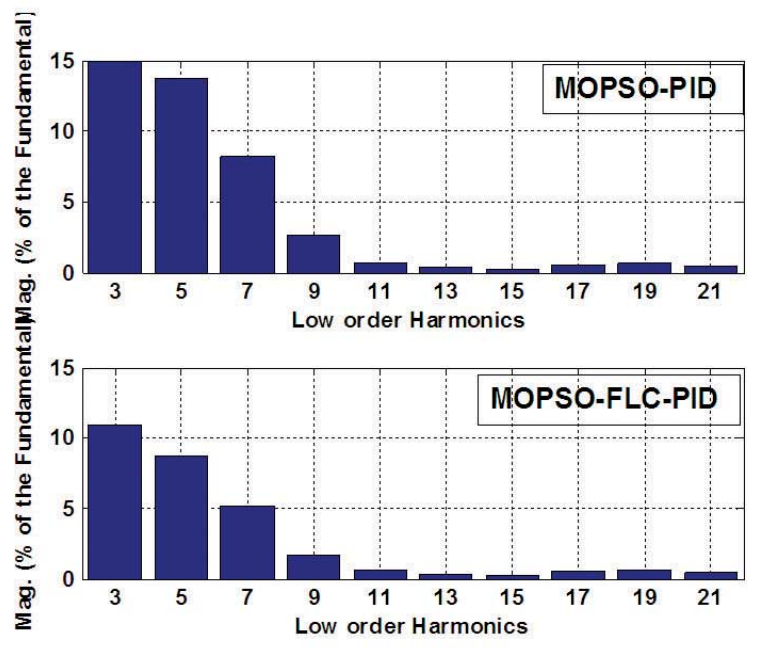

helps in maintaining the dc voltage constant as well as acts as a DC-side active filter and reduces the oscillations in the generator torque which occur due to unbalanced load. The performances comparison, with the SOPSO for tuning fuzzy PID controllers, standard MOPSO-based for tuning PID controller, SOPSO-based for tuning PID controller and Ziegler-Nichols PID approaches, shows the efficiency and superiority of the proposed MOPSO-based fuzzy PID controllers approach in terms of the obtained solution qualities, 
the convergence speed and the simple software implementation of its algorithm. MATLAB/Simulink simulation results show that the proposed design approach is efficient to find the optimal parameters of the FLC-based PID controllers and improves the transient performance of the wave energy system over a wide range of operating conditions. The simulation results demonstrate that the performance of the controllers is satisfactory under steady state as well as dynamic conditions and under balanced as well as unbalanced load conditions. THD levels of the converter output voltage has been estimated using fast Fourier transform which satisfies the IEEE 519-1992 standard. The hybrid generating system with the proposed control strategy is suitable for a small scale standalone variable speed wave turbine installation for remote area power supply. Moreover, the proposed technique can be extended to systems with different topologies.

\section{References}

[1] D. Halamay, T. Brekken, A. Simmons, and S.McArthur, "Reserve requirement impacts of large-scale integration of wind, solar and ocean wave power generation,” IEEE Trans. Sustain. Energy. 2011; 2(3): 321-328. http://dx.doi.org/10.1109/TSTE.2011.2114902

[2] F. Fusco and J. Ringwood, "Variability reduction through combination of wind and waves: A Irish case study," Energy. 2010; 35: 314-325, 2010. http://dx.doi.org/10.1016/j.energy.2009.09.023

[3] X. Yang, Y. Song, G. Wang, and M. Wang, "A comprehensive review on the development of sustainable energy strategy and implementation in China," IEEE Trans. Sustain. Energy. 2010; 1(2): 57-65. http://dx.doi.org/10.1109/TSTE.2010.2051464

[4] Li Wang and Zan-Jia Chen "Stability Analysis of a Wave-Energy Conversion System Containing a Grid-Connected Induction Generator Driven by a Wells Turbine" IEEE Transactions On Energy Conversion. JUNE 2010; 25(2): 555-63

[5] Modesto Amundarain, Mikel Alberdi, Aitor J. Garrido, and Izaskun Garrido, "Modeling and Simulation of Wave Energy Generation Plants: Output Power Control" IEEE Transactions On Industrial Electronics. 2011; 58(1): 105-117

[6] S. Benelghali, M.E.H. Benbouzid and J.F. Charpentier, "Marine tidal current electric power generation technology: State of the art and current status," in Proceedings of the 2007 IEEE IEMDC, Antalya (Turkey). May 2007; 2: 1407-12.

[7] Francesco Fusco and John V. Ringwood, "A Simple and Effective Real-Time Controller for Wave Energy Converters" IEEE Transactions On Sustainable Energy. JANUARY 2013; 4(1): 21-30

[8] Bhim Singh and Gaurav Kumar Kasal, "Voltage and Frequency Controller for a three-phase four wire autonomous wind energy conversion systems," IEEE Trans. Energy Conversion. 2008; 23(2): 509-518. http://dx.doi.org/10.1109/TEC.2008.918620

[9] Bhim Singh and Gaurav Kumar Kasal, "Solid-State Voltage and Frequency Controller for a stand alone wind power generating system," IEEE Trans. Power Electronics. 2008; 23(3): 1170-1177. http://dx.doi.org/10.1109/TPEL.2008.921190

[10] G. Iwanski and W. Koczara, "The DFIG-based power generation system with UPS function for variable-speed applications," IEEE Trans. Ind. Electron. Aug. 2008; 55(8): 3047-54. http://dx.doi.org/10.1109/TIE.2008.918473

[11] J. $\mathrm{Hu}, \mathrm{Y} . \mathrm{He}, \mathrm{L} . \mathrm{Xu}$, and B. W. Williams, "Improved control of DFIG systems during network unbalance using PI-R current regulators," IEEE Trans. Ind. Electron. 2009; 56(2): 439-451. http://dx.doi.org/10.1109/TIE.2008.2006952

[12] Bhim Singh and Jitendra Solanki, "A solid-state compensator with energy storage for isolated diesel-generator set," in Proc. of IEEE International Symposium on Industrial Electronics, Canada. 2006: 1774-1778

[13] L.M.Fernandez, J.R.Saenz and F.Jurado, "dynamic Models of wind pharms with fixed speed wind turbines," Renewable Energy. 2006; 31(8): 1203-1230. http://dx.doi.org/10.1016/j.renene.2005.06.011

[14] Karbakhsh, M. ; Abutorabi, H. ; Khazaee, A. "An Enhanced MPPT Fuzzy Control of a Wind Turbine Equipped with Permanent Magnet
Synchronous Generator" 2nd International e-Conference on Computer and Knowledge Engineering (ICCKE). 2012: 77 - 82.

[15] H. Li, Z. Chen, "Overview of different wind generator systems and their comparisons," IET Renewable Power Generation. 2007; 2(2): 123-138. http://dx.doi.org/10.1049/iet-rpg:20070044

[16] Md. Enamul Haque, Michael Negnevitsky, and Kashem M. Muttaqi, "A Novel Control Strategy for a Variable-Speed Wind Turbine With a Permanent-Magnet Synchronous Generator" IEEE Transactions On Industry Applications, Vol. 46, No. 1, January/February 2010 331. http://dx.doi.org/10.1109/TIA.2009.2036550

[17] T. F. Chan and L. L. Lai, "Permanent-magnet machines for distributed generation: A review," in Proc. IEEE Power Eng. Annu. Meeting. 2007: 1-6.

[18] C. N. Bhende, S. Mishra, Senior Member, IEEE, and Siva Ganesh Malla "Permanent Magnet Synchronous Generator-Based Standalone Wind Energy Supply System" IEEE Transactions On Sustainable Energy. October 2011; 2(4): 361 - 373.

[19] Mohamed .M. Ismail “ Adaptation of PID Controller using AI Techniques for Speed Control of Isolated Steam Turbine" International Journal Of Control, Automation And Systems. January 2012; 1(1): 24-30.

[20] Zadeh, L. A. Fuzzy sets, Information Control. 1965; 8: 339-353. http://dx.doi.org/10.1016/S0019-9958(65)90241-X

[21] Hassen T. Dorrah, Ahmed M. El-Garhy, Mohamed E. El-Shimy "Design of PSO-Based Optimal Fuzzy PID Controllers for the TwoCoupled Distillation Column Process" Proceedings of the 14th International Middle East Power Systems Conference (MEPCON'10), Cairo University, Egypt, December 19-21, 2010,

[22] Ching-Chang Wong and Shyuan-Ming Feng, "Switching-type fuzzy controller design by genetic algorithms," Fuzzy Sets and Systems. 1995; 74: 175-185, 1995. http://dx.doi.org/10.1016/01650114(94)00359-F

[23] Chul-Hwan Jung, Chang-Shik Ham, and Kuhn-II Lee, "A real-time self-tuning fuzzy controller through scaling factor adjustment for the steam generator of NPP,' Fuzzy Sets and Systems. 1995; 74: 53-60. http://dx.doi.org/10.1016/0165-0114(95)00035-J

[24] Huiwen Deng and Yi Wang. "An adaptive fuzzy logic controller with self-tuning scaling factors based on neural networks," Autonomous Decentralized Systems, ISADS.Proceedings. April 2005: 392-396

[25] Jing-jing Qin ; Xian-Xia Zhang ; Gui-tao Cao "Particle swarm optimization based scaling factor tuning for 3-D fuzzy logic controller" 2010 Seventh International Conference on Fuzzy Systems and Knowledge Discovery (FSKD). 2010; 2: 711 - 715.

[26] Sawsan Gharghory, Hanan Kamal " Modified PSO for Optimal Tuning of Fuzzy PID Controller" IJCSI International Journal of Computer Science Issues. March 2013; 10(2): 462-471

[27] Dongyun Wang ; Fuling Fan "Parameters Tuning of Fuzzy Controller for Rotated Pendulum Based on Improved Particle Swarm Optimization" International Conference on Computational Intelligence and Software Engineering, 2009. CISE 2009. Page(s): $1-5$

[28] J. Kennedy and R. Eberhart, "Particle swarm optimization" Proceedings, IEEE International Conf. on Neural Networks. 1995; 4: 1942-1948. 
[29] Y. Shi and R. Eberhart, "Empirical study of particle swarm optimization" Proceedings of the 1999 Congress on Evolutionary Computation. 1999; 3.

[30] R. Eberhart and Y Shi. "Particle swarm optimization: developments, applications and resources" Proceedings of the 2001 Congress on
Evolutionary Computation. 2001; 1: $81-86$

[31] Y. Shi and R. Eberhart. "Parameter Selection in Particle Swarm Optimization" Proc. Seventh Annual Conf. on Evolutionary Programming. 1998: 591-601. http://dx.doi.org/10.1007/BFb0040810 PMid:9721156 